\title{
Ultra Sonographic Eveluation and Computed Tomography Ao Abdominal Trauma - A Comparative Study
}

\author{
Divya $\mathrm{Y}^{1}$, Rajkumar $\mathrm{G}^{2}$, Abubacker Sulaiman $\mathrm{F}^{3}$, Lavanya $\mathrm{G}^{4}$, Saravanan $\mathrm{K}^{5}$, Ramya Kalaiarasan ${ }^{6}$ \\ ${ }^{1}$ Final Year Postgraduate, Department of Radio Diagnosis, Chettinad Hospital \& Research Institute, ${ }^{2}$ Senior Resident, \\ Department of Radio Diagnosis, Chettinad Hospital \& Research Institute, ${ }^{3}$ Professor, Department of Radio Diagnosis, \\ Chettinad Hospital \& Research Institute, ${ }^{4}$ Senior Resident, Department of Radio Diagnosis, Chettinad Hospital \& Research \\ Institute, ${ }^{5}$ Senior Resident, Department of Radio Diagnosis, Chettinad Hospital \& Research Institute, ${ }^{6}$ Senior Resident, \\ Department of Radio Diagnosis, Chettinad Hospital \& Research Institute, India
}

Corresponding author: Dr. Rajkumar G, Senior Resident, Department of Radio Diagnosis, Chettinad Hospital \& Research Institute, Kelambakkam, Chennai - 603103. India

DOI: http://dx.doi.org/10.21276/ijcmsr.2019.4.3.59

How to cite this article: Divya Y, Rajkumar G, Abubacker Sulaiman F, Lavanya G, Saravanan K, Ramya Kalaiarasan. Ultra sonographic eveluation and computed tomography Ao abdominal trauma - a comparative study. International Journal of Contemporary Medicine Surgery and Radiology. 2019;4(3):C267-C270.

\section{A B S T R A C T}

Introduction: Trauma is one of the leading causes of death throughout the world. This is due to road traffic accidents, violent assault, wars, and natural calamities. Ultrasound and multi-detector CT scan helps in accurately diagnosing the various injuries, thus helping the clinician to plan for appropriate treatment. Hence, the aim of this study was to assess the diagnostic efficacy between CT and Ultrasound in abdominal trauma patients.

Material and methods: The present study was a prospective study which was conducted in the Department of RadioDiagnosis, Chettinad Hospitals and Research Institute. Total 142 patients with history of abdominal trauma were involved in the study. The selected patients were subjected to screening ultrasound abdomen and contrast enhanced CT abdomen. Results: The results showed that when Ultrasound was compared with CT, it was found that US showed $60 \%$ sensitivity $98 \%$ specificity, 91\% accuracy, and had positive predictive value of $89 \%$ and negative predictive value of $98 \%$. While on the other hand, CT had sensitivity of $96 \%$, specificity of $100 \%$, accuracy of $97 \%$, and positive predictive value of $100 \%$ and negative predictive value of $79 \%$.

Conclusion: CT is the most sensitive and specific diagnostic modality in evaluation of abdominal, thus helping the surgeons plans the line of management accordingly.

Keywords: Abdominal trauma, CT, Ultrasound, Organ Injuries

\section{INTRODUCTION}

Trauma is one of the major diagnostic challenges faced by both surgeons and the radiologist. It is a common cause of death, ranging from 15-50 years in developing countries. Abdominal trauma (AT) is one of the common reasons of death in India. Abdominal injuries account for $10 \%$ of all deaths attributed to trauma. Most of the deaths occur at the scene of accident and result from neurological injuries, hemorrhage and multi-organ failure. ${ }^{1}$

Trauma is the leading cause of death in persons under 45 years of age, with $10 \%$ of these fatalities attributable to abdominal injuries. Missed intra-abdominal injuries continue to cause preventable deaths. Blunt abdominal trauma usually results following road traffic accidents, assaults, recreational accidents, or falls. ${ }^{2}$

Clinical findings are compounded by factors like fractures of ribs, vertebrae and pelvis or other associated injuries. Major change in the paradigm of the management of blunt abdominal trauma is the introduction of non- operative management coinciding with the availability of computed tomography (CT) scans, hence the role of imaging becomes even more paramount for the safe practice of such surgical restraint. $^{3}$

The mortality rate due to trauma has increased significantly second to infectious disease. Abdominal injuries are frequently encountered cause of traumatic deaths, following head and chest trauma. The AT victims are routinely evaluated with physical examination and clinical signs which are inconclusive for effective diagnosis. Particularly, patient having any one or more of the following conditions such as decreased consciousness level, neurological deficit, injuries, drugs and other medications, affect diagnostic efficacy. ${ }^{4,5}$ Diagnostic peritoneal lavage (DPL), Interventional radiology and computed tomography (CT) are used for effective abdominal evaluation in trauma. Among these diagnostic tests, ultrasound (FAST) Focused Assessment with Sonography for Trauma and computed tomography (CT) are widely used. It is a method to detect intra-peritoneal fluid in an emergency setting USG is a non-invasive, does not use ionizing radiation, less expensive and can be performed in 
the emergency department, concurrently with other aspects of resuscitation. ${ }^{6}$

Computed tomography is the imaging modality of choice to evaluate hemodynamic stable patients. It is sensitive (9297.6\%) and specific (98.7\%). The main advantage is the ability to detect arterial contrast extravasation, uncontained or as a pseudoaneurysm, which predicts the need for surgery or angioembolisation. ${ }^{7}$

CT also accurately evaluates the retro-peritoneum, but it is less sensitive for detecting hollow viscous injuries, although detection rates are improving with increasing experience. It is also the modality of choice for diagnosing injuries to the diaphragm, which may result in major morbidity and mortality if undetected and may not present until many years after the event.

Interventional radiology plays an increasingly important role in planning and management of injuries. Transcatheter arterial embolization may serve as an alternative to uncontrolled bleeding in the upper abdomen and is the preferred treatment in the pelvis and the retro-peritoneum. ${ }^{8,9}$ The use of CT has been advocated for evaluation of occult intra-abdominal trauma in patients undergoing prolonged anesthesia for repair of other injuries in which serial examinations of the abdomen cannot be performed. Ultrasound offers many advantages. It is portable, noninvasive, and rapid and facilitates serial examinations. It has no known complications and can be used in patients with previous laparotomy, pregnancy, or clotting disorder. However, ultrasound is operator dependent and is technically limited by the presence of obesity, subcutaneous emphysema, or ascites. Furthermore, ultrasound is not sensitive for detection of hollow viscous injuries. ${ }^{10}$ Hence, the aim of the present study was to assess the diagnostic efficacy of US and CT in abdominal trauma patients.

\section{MATERIAL AND METHODS}

The present study was a prospective study which was conducted among 142 patients with traumatic injuries to abdomen who were admitted in Chettinad Medical Hospital and Research Institute from Dec 2016 to Apr 2017. The criteria for selection were patients with traumatic injury to abdomen with or without other injuries. USG scans were performed using "Voluson" machine, with 2.5-5 and 7.5-12 $\mathrm{MHz}$ curvilinear and linear probes. CT scans were performed using "Philips" helical sub second (0.75 Sec) scanner. (Figure no. 1).

Images were reconstructed at $5 \mathrm{~mm}$ intervals using standard reconstruction kernel and 180 degrees linear interpolation. Post-operative findings are recorded, follow up studies have done. In case of deaths, causes of deaths were established by post mortem report. As patient characteristics could influence the accuracy of ultrasound, potential differences in sensitivity between patient groups were evaluated.

In addition, sensitivity and predictive values of ultrasound in attending radiologists including supervised residents were compared with those of unsupervised residents. In addition, sensitivity and predictive values of ultrasound in attending radiologists including supervised residents were compared with those of unsupervised residents.

\section{Contrast Material}

All patients were administered with IV contrast with an automated injector after the initial scout image via a largebore $(18-20 \mathrm{G})$ peripheral venous line. A total of $120 \mathrm{ml}$, $60 \%$ non - ionic iodinated contrast was administered intravenously at a rate of $2-4 \mathrm{ml} / \mathrm{s}$. Scanning was performed 70-90 seconds later following contrast infusion.

\section{Gold standard - Surgery}

Despite the systematic approach and diagnostic superiority of CT, coupled with the use of adjunctive diagnostic methods, exploratory laparotomy remains to be the most accurate method in diagnosing intra-abdominal injury.

\section{STATISTICAL ANALYSIS}

The data analyzed was entered into the excel sheet and inferential statistics was applied using two way ANOVA for comparing USG and CT. p values less than 0.05 was taken to indicate statistically significant differences. All analyses were performed in Prism Graph pad (USA).

\section{RESULTS}

In the present study, there were 107 males and 35 females. The reason for higher incidence in this age group is probably due to a higher mobility and more use of two wheelers and more active and outdoor life. The maximum number of cases was in $3 \mathrm{rd}$ and 4 th decade. The most common age group was 21-30 years followed by 31-40 years. (Graph 1 )

The most common cause of traumatic abdomen is blunt injury is Road traffic accidents amounting to $60 \%$ followed by fall of wall on abdomen among $20 \%$ patients. Various organ injuries such as free fluid followed by spleen, liver and

\begin{tabular}{|l|c|c|}
\hline Parameters & USG & CT \\
\hline Sensitivity & 59.37 & 95.97 \\
\hline Specificity & 98.12 & 100 \\
\hline Accuracy & 91.81 & 97 \\
\hline Positive Predictive Value & 88.93 & 100 \\
\hline Negative Predictive Value & 97.77 & 78.35 \\
\hline
\end{tabular}

Table-1: Shows the comparison between USG and CT based on sensitivity and specificity

\begin{tabular}{|l|c|c|c|c|}
\hline Row Factor & USG & CT & Difference & $\mathbf{9 5 \%}$ Cl \\
\hline Free fluid & 100 & 121 & 21 & -39.83 to 81.83 \\
\hline Liver & 32 & 64 & 32 & -28.83 to 92.83 \\
\hline Spleen & 35 & 70 & 35 & -25.83 to 95.83 \\
\hline Kidneys & 13 & 32 & 19 & -41.83 to 79.83 \\
\hline Bladder & 3 & 4 & 1 & -59.83 to 61.83 \\
\hline Others & 0 & 2 & 2 & -58.83 to 62.83 \\
\hline Table-2: Shows the comparison based on various organ inju- \\
ries \\
\hline
\end{tabular}

\begin{tabular}{|l|c|c|c|c|}
\hline Source of Variation & Df & Sum-of-squares & $\begin{array}{c}\text { Mean } \\
\text { square }\end{array}$ & F \\
\hline Column Factor & 1 & 1008 & 1008 & 9.702 \\
\hline Row Factor & 5 & 16699 & 3340 & 32.13 \\
\hline
\end{tabular}

Table-3: Shows the difference between column factor and row factor 


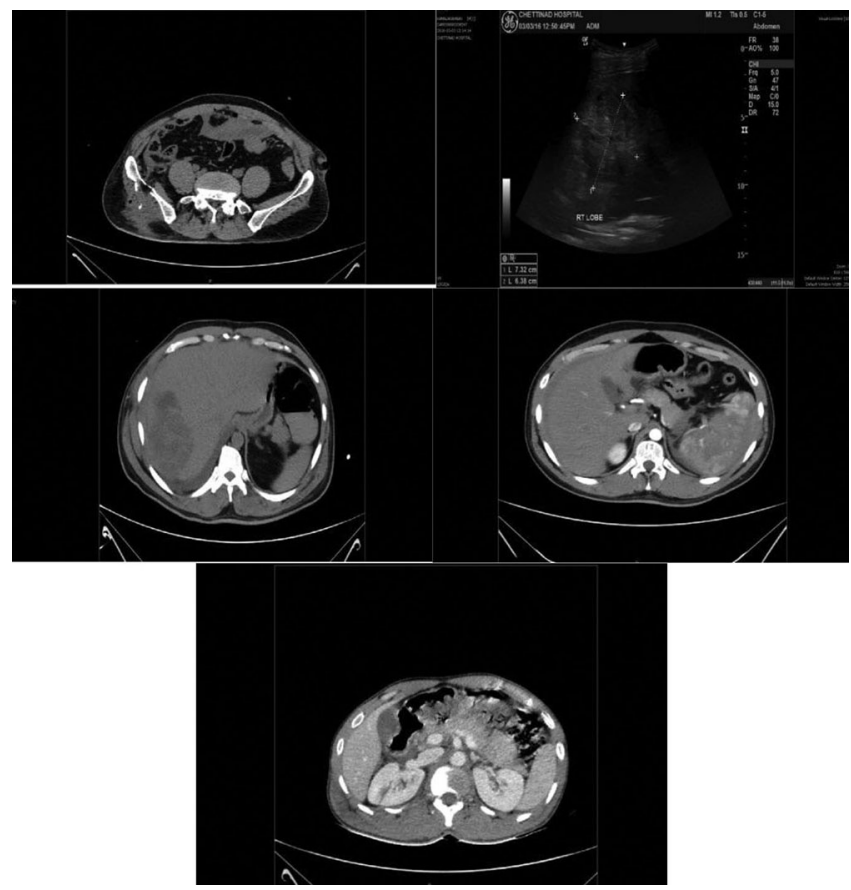

Figure-1: Shows the types of images. Image 1 shows NECT in a case of Gunshot Injury Abdomen demonstrating entry wound near the Left Iliac region. Image 2 shows ultrasound abdomen with hyper-echoic focus in the right lobe of liver. Image 3 shows NECT of the liver contusion showing an ill hypo-dense defined focus seen in right lobe of liver. Image 4 shows enhanced CT was done for the same patient, showing Heterogeneous enhancement of the splenic parenchyma, suggestive of parenchymal hemorrhage (AAST Grade IIIIV). Image 5 shows the contrast Enhanced CT done on the same patient showed Right renal laceration (AAST Grade III Injury).

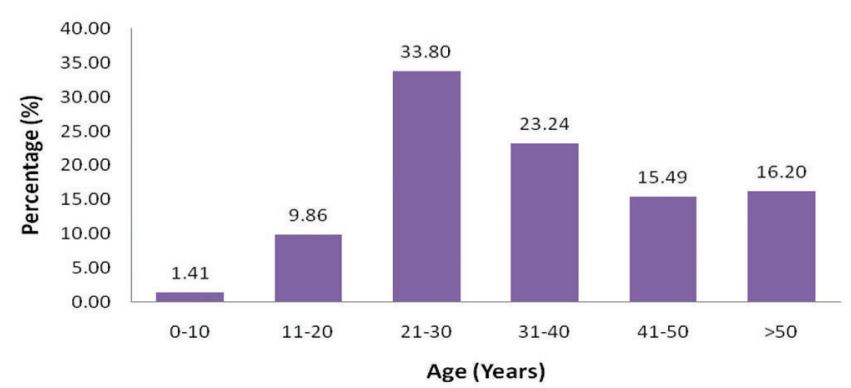

Graph-1: Shows the distribution of data based on age groups

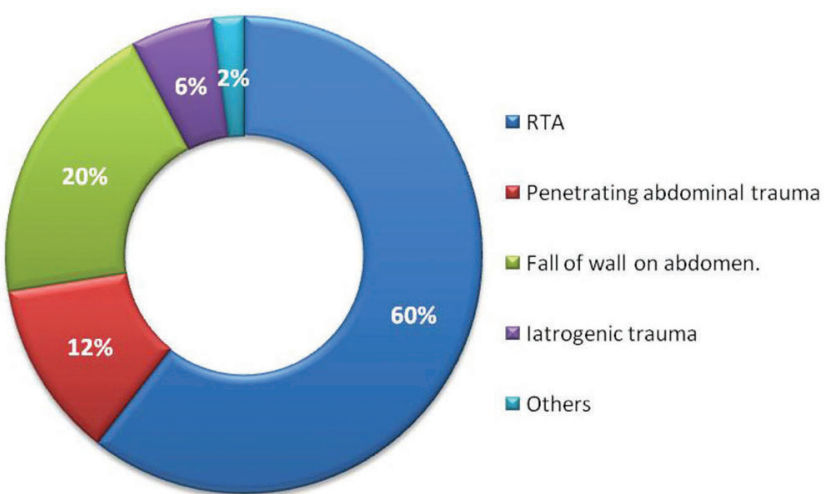

Graph-2: Shows the distribution of data based on mode of trauma

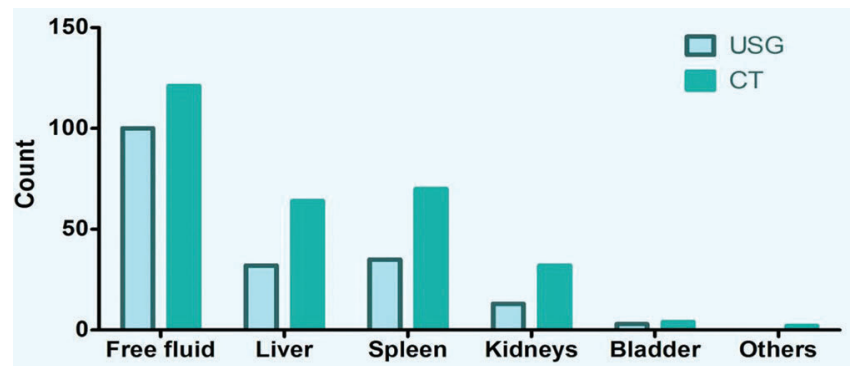

Graph-3: Shows the diagnostic comparison of CT and USG in various organ injuries

kidney were better detected with $\mathrm{CT}$ when compared with US. (Graph 2)

Specificity, sensitivity, accuracy followed by positive and negative predictive value was more with $\mathrm{CT}$ in comparison to ultrasound. The row factor was higher in $\mathrm{CT}$ when compared with US (Table 1 and 2). The column factor and row factor with $\mathrm{F}$ value was found to be 9.702 and 32.13. (Table 3).

\section{DISCUSSION}

In the current study, among 142 cases, free fluid was detected in 100 patients with USG and 121 cases with CT. Liver injury was diagnosed in 32 patients using ultrasound, whereas CT detected 64 cases. About 35 cases showed splenic injury in USG, while CT picked up 70 cases. In case of kidney injury, about 13 cases were picked up in USG, while CT was diagnosed in 32 cases. About 3 cases of bladder injury were diagnosed using USG, and CT again outnumbered with 4 cases and 2 other cases with bowel injury and muscle hematoma, which were abruptly missed on USG, were diagnosed with $\mathrm{CT}$.

Though ultrasound is the best initial imaging modality of choice, many injuries were missed, when used alone. While USG abdomen was used in conjunction with $\mathrm{CT}$ abdomen, better diagnostic results were obtained. Certain Injuries that were missed on USG were accurately diagnosed with CT.

The authors of many studies have compared CT and ultrasound in the evaluation of blunt abdominal trauma. Rothlin et al prospectively studied 312 patients with blunt trauma and confirmed sonographic results with the use of $\mathrm{CT}$ in all patients with intra-abdominal fluid on the initial ultrasound examination and in $25 \%$ of patients without signs of hemoperitoneum on the initial study. Serial sonograms were performed in patients with minimal intra-abdominal fluid or negative initial findings. ${ }^{11,12,13}$

However contraindications are very few, although patients co-operation is essential the patients with rib fractures may present problems, subcutaneous emphysema may affect both the ability to perform the study and quality of scan.

On comparing Ultrasound with CT, USG was around $60 \%$ sensitive $98 \%$ specific, $91 \%$ accurate, and had positive predictive value of $89 \%$ and Negative predictive value of $98 \%$. While on the other hand, CT had sensitivity of $96 \%$, specificity of $100 \%$, accuracy of $97 \%$, and positive predictive value of $100 \%$ and negative predictive value of $79 \%$. In the present study, CT has a greater imaging modality in the diagnosis of abdominal trauma.

Although CT is the Gold standard imaging modality in the 
evaluation of blunt injury abdomen, combined usage of USG and CT abdomen yields better and accurate diagnosis.

Diagnostic imaging is widely used in the work-up of patients with acute abdominal pain. Ultrasound and computed tomography (CT) are both frequently used on top of clinical and laboratory evaluation. The American College of Radiology suggests an abdomen/pelvis CT with contrast medium in patients with acute abdominal pain. ${ }^{14}$

Ultrasound accuracy could also be lower in specific patient subgroups, such as in obese patients, women, and in specific age groups, especially women of reproductive age. CT, on the other hand has good inter-observer agreement in general, and even excellent inter-observer agreement for frequent diagnoses causing acute abdominal pain (e.g. appendicitis and diverticulitis). ${ }^{15,16}$ In the present study, limitations were related to the lobulations, congenital clefts, and prominent left hepatic lobe and streak motion artifacts. The differentiation of subcapsular from pericapsular peritoneal bleeding may be difficult. Inhomogeneous contrast enhancement, especially with fast dynamic or spiral scanning may produce diagnostic difficulties.

\section{CONCLUSION}

Ultrasonography is valuable initial diagnostic modality in patient with abdominal trauma whereas computed tomography is required in most positive cases of ultrasound to delineate the exact extension of injury and to find out other significant injuries. CT should be used in patients with symptomatic and or in patients with unexplained findings even with the negative USG findings. Hence, CT has got more sensitivity and specificity in imaging modalities and for the management approach.

\section{REFERENCES}

1. Weishaupt D, Grozaj AM, Willmann JK, et al. Traumatic injuries: imaging of abdominal and pelvic injuries. Eur Radiol 2002;12(1):1295-1311.

2. Wolfman NT, Bechtold RE, Scharling ES, et al. Blunt upper abdominal trauma: evaluation by CT. AJR Am J Roentgenol 1992;158(3): 493.

3. Hamidi MI, Aldaoud KM, Qtaish I. The role of Computed Tomography in Blunt Abdominal Trauma. Sultan Qaboos Univ Med J 2007; 7(1): 41-6.

4. Poletti PA, Wintermark M, Schnyder P, et al. Traumatic injuries: role of imaging in the management of the polytrauma victim (conservative expectation). Eur Radiol 2002;12(6):969.

5. Yale-Loehr AJ, Kramer SS, Quinlan DM, et al. CT of severe renal trauma in children: evaluation and course of healing with conservative therapy. AJR Am J Roentgenol 1989;152(3): 109.

6. Sanjeev Suman, Babita, G.N. Singh. Study on abdominal trauma patients comparatively by ultrasonography and CT. International Journal of Contemporary Medical Research 2017; 4(4):872-873.

7. Wing VW, Federle MP, Morris JA, et al. The clinical impact of CT for blunt abdominal trauma. AJR Am J Roentgenol 1985;145(3): 11.

8. Laméris W, van Randen A, Bipat S, Bossuyt PM, Boermeester MA, Stoker J. Graded compression ultrasonography and computed tomography in acute colonic diverticulitis: meta-analysis of test accuracy. Eur Radiol 2008; 18(1):2498-2511.

9. Judy Mary Kurian, Ganesh K, Praveen Kumar John, Prasad Hegde, Chidananda Murthy, Arun Kumar. A comparative evaluation of USG and MRCP findings in biliary and pancreatic pathologies. International Journal of Contemporary Medical Research 2017; 4(1):212215.

10. Rodriguez C, Barone JE, Wilbanks TO, Rha CK, Miller K. Isolated free fluid on computed tomographic scan in blunt abdominal trauma: a systematic review of incidence and management. J Trauma 2002; 53(1):7985 .

11. Rothlin MA, Naf RN, Amgwerd M, et al. Ultrasound in blunt abdominal and thoracic trauma. J Trauma 1993; 34(6):488-495.

12. Huang MS, Liu M, Wu JK, et al: Ultrasonography for the evaluation of hemoperitoneum during resuscitation: A simple scoring system. J Trauma 1994; 36(5):173-177.

13. Mallik K, Vashisht S, Thakur S, Srivastava DN. Comparative evaluation of ultrasonography and $\mathrm{CT}$ in patients with abdominal trauma: A prospective study. Indian J Radiol Imaging. 2000; 10(4):237-43.

14. Terasawa T, Blackmore CC, Bent S, Kohlwes RJ. Systematic review: computed tomography and ultrasonography to detect acute appendicitis in adults and adolescents. Ann Intern Med 2004; 141(2):537546.

15. Yao DC, Jeffrey RB, Mirvis SE, Weekes A, Federle MP, Kim C, et al. using contrast-enhanced helical CT to visualize arterial extravasation after blunt abdominal trauma: incidence and organ distribution. AJR Am J Roentgenol 2002; 178(3):17-20.

16. Radwan MM, Abu-Zidan FM. Focussed Assessment Sonography Trauma (FAST) and CT scan in blunt abdominal trauma: surgeon's perspective. Afr Health Sci. 2006; 6(3):187-190.

Source of Support: Nil; Conflict of Interest: None

Submitted: 23-07-2019; Accepted: 19-08-2019; Published online: 25-09-2019 